\title{
Improvement of the Lipid Profile with Zofenopril in Hypertensive Patients with the Metabolic Syndrome
}

\author{
Evangelos C. Rizos, Sofia Tsouli, Michalis Doumas, Michael Kostapanos, Konstantinos Lagos, \\ Alexandros D. Tselepis and Moses Elisaf*
}

Department of Internal Medicine, Medical School, University of Ioannina, 45110 Ioannina, Greece

\begin{abstract}
The effect of the anti-hypertensive drug zofenopril on metabolic parameters in patients with the metabolic syndrome (MetS) is currently unknown. The present study was conducted in order to evaluate whether zofenopril affects parameters involved in the atherosclerotic process. This open-label intervention study included 60 adult patients with essential hypertension that additionally had 2 or more of the following MetS criteria: waist circumference $>102 \mathrm{~cm}$ in men and $>88 \mathrm{~cm}$ in women, triglycerides $>150 \mathrm{mg} / \mathrm{dl}$, high density lipoprotein cholesterol (HDL-C) $<40 \mathrm{mg} / \mathrm{dl}$ in men and $<50$ $\mathrm{mg} / \mathrm{dl}$ in women, and fasting glucose $\geq 100 \mathrm{mg} / \mathrm{dl}$. Zofenopril reduced systolic blood pressure by $11 \%(\mathrm{p}<0.001)$ and diastolic blood pressure by $7 \%(\mathrm{p}=0.004)$. Zofenopril also decreased triglycerides by $24 \%(\mathrm{p}=0.05)$ and apolipoprotein B levels by $7 \%(\mathrm{p}=0.04)$. In addition, the drug improved the lipid profile by decreasing both small dense low density lipoprotein (LDL) cholesterol by $22 \%(\mathrm{p}=0.04)$, as well as the proportion of the cholesterol of small dense LDL subfraction by $8 \%(\mathrm{p}=0.04)$, whereas it significantly increased the large LDL cholesterol by $6 \%(\mathrm{p}=0.05)$ and the proportion of the cholesterol of large LDL subfraction by $7 \%(\mathrm{p}=0.05)$. Accordingly, mean LDL particle size was increased by $0.04 \%(\mathrm{p}=0.05)$ with zofenopril. The reduction of small dense LDL subfractions was significantly correlated with the reduction of ApoB levels, even after correction for baseline values (Pearson correlation coefficient $0.8, \mathrm{p}=0.03$ ), and with the reduction of triglycerides (Spearman correlation coefficient 0.77, $\mathrm{p}=0.02$ ). An additional effect of zofenopril was the reduction of the enzymatic activity of lipoprotein-associated phospholipase $\mathrm{A}_{2}$ (Lp-PLA2) by $9 \%$ ( $\mathrm{p}=0.05$ ). On the other hand, zofenopril did not influence glucose homeostasis and fibrinogen levels. In conclusion, zofenopril is an effective anti-hypertensive agent with additional favourable effects on the atherogenic dyslipidemia of MetS.
\end{abstract}

Keywords: Zofenopril, hypertension, metabolic syndrome, glucose, cholesterol, lipoprotein associated phospholipase A2, small dense LDL.

\section{INTRODUCTION}

The metabolic syndrome (MetS) is characterized by 3 or more of the following criteria, according to the updated AHA NHLBI statement: waist circumference $>102 \mathrm{~cm}$ in men and $>88 \mathrm{~cm}$ in women, triglycerides $>150 \mathrm{mg} / \mathrm{dl}$, high density lipoprotein cholesterol (HDL-C) $<40 \mathrm{mg} / \mathrm{dl}$ in men and $<50 \mathrm{mg} / \mathrm{dl}$ in women, blood pressure $\geq 130 / 85 \mathrm{~mm} \mathrm{Hg}$ and fasting glucose $\geq 100 \mathrm{mg} / \mathrm{dl}$ [1]. The importance of MetS is continuously growing, taking into account that in the United States $25 \%$ of adults older than 20 years and $45 \%$ older than 50 years are affected by this syndrome, while in Greece $25 \%$ of men and $15 \%$ of women older than 18 years meet the MetS criteria [2-4]. Patients with MetS are at increased risk for type 2 diabetes mellitus and cardiovascular disease $[1,5-8]$ and a recent meta-analysis showed that these patients have a relative risk (RR) of 1.78 for cardiovascular events and death [6].

Zofenopril is an angiotensin converting enzyme (ACE) inhibitor approved for the treatment of essential hypertension with particular benefit in patients with acute anterior myocardial infarction [9]. The existence of sulfydryl (SH) group

*Address correspondence to this author at the Department of Internal Medicine, Medical School, University of Ioannina, 45110 Ioannina, Greece; Tel: +302651097509; Fax: +302651097016; E-mail: egepi@cc.uoi.gr in zofenopril distinguishes it from the majority of ACE inhibitors and experimental studies suggest that $\mathrm{SH}$ group confers additional properties other than ACE inhibition, such as free radical scavenging effects, and an improvement of endothelial dysfunction $[10,11]$. These effects could be important in patients with MetS, where vascular damage is considered to be related not only to the lipid profile per se, but also to the oxidative damage of vessels, the inflammatory process, and the altered glucose homeostasis $[1,2,4,12,13]$.

The present study was conducted in order to evaluate whether zofenopril affects any of the parameters involved in atherogenenesis beyond its antihypertensive action, in hypertensive patients with MetS.

\section{PATIENTS AND METHODS}

\section{Participants}

This open-label intervention study was conducted in patients attending the Metabolism and Lipid Clinic of the University Hospital in Ioannina, Greece. Caucasian adult patients newly diagnosed with essential hypertension defined as systolic blood pressure (SBP) > $140 \mathrm{~mm} \mathrm{Hg}$ and/or diastolic blood pressure (DBP) $>90 \mathrm{~mm} \mathrm{Hg}$. In addition, patients had 2 or more of the following criteria for MetS: waist circumference $>102 \mathrm{~cm}$ in men and $>88 \mathrm{~cm}$ in women, triglycerides $>150 \mathrm{mg} / \mathrm{dl}$, HDL-C $<40 \mathrm{mg} / \mathrm{dl}$ in men and < $50 \mathrm{mg} / \mathrm{dl}$ in women and fasting glucose $\geq 100 \mathrm{mg} / \mathrm{dl}$. Patients 
with liver or renal disease (serum aminotransferase activity greater than three-fold the upper limit of normal, e.g. 120 IU/l, serum creatinine levels $>1.5 \mathrm{mg} / \mathrm{dl}$, respectively), hypothyroidism (Thyroid stimulation hormone $>5 \mathrm{mIU} / \mathrm{ml}$ ), heavy alcohol consumption ( $>3$ drinks per day), or patients taking drugs that could affect lipid metabolism, as well as renal or hepatic function were excluded. In addition, the study population did not take any other antihypertensive drugs.

There were no differences in diet composition of the patients throughout the study. The daily distribution of nutrients during the study was: carbohydrates $52.5 \pm 4.1 \%$, fat $28.5 \pm 3.2 \%$ (monounsaturated $15.3 \pm 1.7 \%$, polyunsaturated $7.1 \pm 1.4 \%$ and saturated fatty acids $6.0 \pm 1.1 \%$ ) and protein $19.0 \pm 1.4 \%$.

The Ethical Committee approved the study and all participants gave a formal consent. All patients $(n=64)$ were initially advised for lifestyle modification and zofenopril 30 $\mathrm{mg}$ once daily was administered in those patients who remained hypertensive after a period of 8 weeks $(n=60)$.

\section{Measurements}

All laboratory determinations were carried out after an overnight fast at the beginning of the study and 12 weeks following zofenopril treatment. Serum levels of total cholesterol (TC), HDL-C, triglycerides and glucose were determined with standard automated methods on the Olympus AU600 Clinical Chemistry analyzer (Olympus Diagnostica, Hamburg, Germany). Serum apolipoprotein A1, B and E levels were measured with a Behring Nephelometer BN100 and reagents from Dade Behring Holding GmbH (Liederbach, Germany). Serum insulin levels were measured by an AxSYM insulin assay microparticle enzyme immunoassay on an AxSYM analyzer (Abbott Diagnostics). Fibrinogen was measured by Dade Behring BCS analyzer. Low density lipoprotein cholesterol (LDL-C) was calculated with the Friedewald formula [TC - HDL-C - (TRG /5)], insulin sensitivity with the homeostasis model assessment (HOMA) index ([fasting insulin $(\mathrm{mU} / \mathrm{L}) \mathrm{X}$ fasting glucose $(\mathrm{mg} / \mathrm{dl})]$ / 405), and body mass index (BMI) was estimated as weight $(\mathrm{kg}) /$ height $^{2}\left(\mathrm{~m}^{2}\right)$.

Lipoprotein-associated phospholipase $\mathrm{A}_{2}\left(\mathrm{Lp}-\mathrm{PLA}_{2}\right)$ activity in total plasma was determined by the trichloroacetic acid precipitation procedure using 1-0-hexadecyl-2-[3Hacetyl]-sn-glycero-3-phosphocholine $([3 \mathrm{H}]$ - platelet activating factor [PAF] $)(100 \mu \mathrm{M}$ final concentration) as a substrate [14]. Lp-PLA 2 activity was expressed as nmol PAF degraded per min per ml of plasma.

LDL subclass analysis was performed electrophoretically by using high-resolution 3\% polyacrylamide gel tubes and the Lipoprint LDL System (Quantimetrix). In this method very low density lipoprotein remains at the origin [retention factor $(\mathrm{Rf}=0.0)]$, whereas HDL migrates at the front $(\mathrm{Rf}$ $=1.0$ ). In between several bands can be detected: MID bands $\mathrm{C}, \mathrm{B}$ and $\mathrm{A}$, which correspond mainly to intermediate density lipoprotein (IDL), as well as up to 7 LDL bands. The LDL1 and LDL2 bands correspond to large, buoyant LDL particles, whereas bands LDL3 to LDL7 correspond to small dense LDL particles. The cholesterol content (in $\mathrm{mg} / \mathrm{dl}$ ) of each lipoprotein subfraction, the mean LDL particle size (in A), and the proportion (\%) of the cholesterol of small dense
LDL subfractions (LDL3 to LDL7) over the total LDL-C were determined.

\section{Statistical Analysis}

Descriptive statistics for continuous variables expressed as means with standard deviation (SD), or median with interquartile range were used. Continuous variables were tested for lack of normality with the Kolmogorov-Smirnov test and, accordingly, comparison of the mean values before and after zofenopril treatment was performed with the paired t-test, while comparison of the median values was assessed with the non-parametric Wilcoxon signed rank test. Pearson's (parametric) or Spearman's (non-parametric) tests for correlation were used and partial correlation taking into account the baseline values was additionally performed.

Statistical analysis was done with SPSS 13.0 (SPSS Inc., Chicago, IL). Significance was defined as $\mathrm{p}<0.05$.

\section{RESULTS}

Sixty hypertensive patients ( 26 females) who fulfilled the criteria for MetS were included in the study. The mean age of the patients was 55 years. Most of them were overweight, 3 had type II diabetes mellitus without anti-diabetic medication, and 27 were smokers (Table 1). Both the weight and the waist circumference of the patients remained stable between the beginning and the finalisation of the study, and no major differences in their diet were noticed.

Table 1. Baseline Characteristics of Study Patients

\begin{tabular}{|c|c|}
\hline $\mathrm{N}$ (females $/$ males) & $60(26 / 34)$ \\
\hline Age (years) & $55 \pm 11$ \\
\hline Weight $(\mathrm{kg})$ & $79 \pm 14$ \\
\hline BMI $\left(\mathrm{kg} / \mathrm{m}^{2}\right)$ & $28.9 \pm 4.9$ \\
\hline Smokers & 27 \\
\hline Diabetes & 3 \\
\hline
\end{tabular}

- $\quad$ BMI: body mass index.

- Mean values \pm standard deviation.

Zofenopril reduced SBP by $11 \%(\mathrm{p}<0.001)$ and DBP by $7 \%(\mathrm{p}=0.004)$ (Table 2). Zofenopril also decreased triglycerides by $24 \%(\mathrm{p}=0.05)$, and apolipoprotein $\mathrm{B}$ levels by $7 \%$ $(\mathrm{p}=0.04)$. In addition, zofenopril improved the lipid profile by decreasing both small dense LDL cholesterol by $22 \%$ $(\mathrm{p}=0.04)$, as well as the proportion of the cholesterol of small dense LDL subfraction by $8 \%(\mathrm{p}=0.04)$, whereas it significantly increased the large LDL cholesterol by $6 \%(\mathrm{p}=0.05)$, and the proportion of the cholesterol of large LDL subfraction by $7 \%(\mathrm{p}=0.05)$. Accordingly, mean LDL particle size was slightly increased following zofenopril treatment $(\mathrm{p}=0.05)$.

The reduction of small dense LDL subfractions was significantly correlated with the reduction in apolipoprotein B levels, even after correction for baseline values (partial correlation), with a Pearson correlation coefficient (r) 0.8 $(\mathrm{p}=0.03)$. Moreover, the reduction of small dense LDL subfractions was correlated with the reduction in triglycerides (Spearman correlation coefficient $0.77, \mathrm{p}=0.02$ ). 
Table 2. Serum Metabolic Parameters at Baseline and 12 Weeks Following Zofenopril Treatment

\begin{tabular}{|c|c|c|c|}
\hline & Baseline* & 12 weeks* & $\mathbf{P}^{* *}$ \\
\hline $\mathrm{SBP}(\mathrm{mmHg})$ & $150 \pm 10$ & $133 \pm 12$ & $<0.001$ \\
\hline $\mathrm{TC}(\mathrm{mg} / \mathrm{dL})$ & $230 \pm 52$ & $220 \pm 37$ & NS \\
\hline Triglycerides (mg/dL) & $196(154-232)$ & $149(119-274)$ & 0.05 \\
\hline LDL-C (mg/dL) & $144 \pm 41$ & $136 \pm 33$ & NS \\
\hline ApoA1 (mg/dL) & $142 \pm 26$ & $140 \pm 17$ & NS \\
\hline ApoB (mg/dL) & $118 \pm 29$ & $110 \pm 24$ & 0.04 \\
\hline ApoE (mg/L) & $5.1 \pm 1.9$ & $4.8 \pm 1.3$ & NS \\
\hline Insulin $(\mathrm{mU} / \mathrm{L})$ & $9.7 \pm 3.3$ & $11.0 \pm 8.2$ & NS \\
\hline HOMA index & $2.5 \pm 0.9$ & $2.9 \pm 0.9$ & NS \\
\hline Fibrinogen (mg/dL) & $394 \pm 95$ & $375 \pm 81$ & NS \\
\hline Small dense LDL cholesterol(mg/dL) & $32 \pm 12$ & $25 \pm 12$ & 0.04 \\
\hline Large LDL cholesterol (mg/dL) & $109 \pm 26$ & $115 \pm 26$ & 0.05 \\
\hline Small dense LDL (\%) & $20 \pm 7$ & $12 \pm 6$ & 0.04 \\
\hline Large LDL (\%) & $81 \pm 7$ & $88 \pm 8$ & 0.05 \\
\hline
\end{tabular}

SBP: systolic blood pressure; DBP: diastolic blood pressure; TC: total cholesterol; HDL-C: high density lipoprotein cholesterol; LDL-C: low density lipoprotein cholesterol; ApoA1: apolipoprotein A1; ApoB: apolipoprotein B; ApoE: apolipoprotein E; Lp(a): lipoprotein (a); HOMA: homeostasis model assessment index; Lp-PLA2: lipoprotein-associated phospholipase A2.

* Mean values \pm standard deviation or median with interquartile range.

**NS: not significant.

An additional effect of zofenopril was the reduction of the enzymatic activity of Lp-PLA 2 by $9 \%(\mathrm{p}=0.05)$. In contrast, zofenopril did not influence glucose homeostasis, as this was estimated with glucose, insulin levels and insulin sensitivity with the HOMA index. Finally, zofenopril had no effect on fibrinogen levels (Table 2).

\section{DISCUSSION}

In the present study, we found that zofenopril exerts additional benefit beyond its anti-hypertensive action in patients with MetS. This was evident by the reduction in apolipoprotein B-containing lipoprotein particles. In addition, zofenopril improved the lipid profile towards a preponderance of large LDL subfractions over small dense LDL subfractions. Thus, any potential effect of zofenopril on morbidity/mortality in patients with MetS may be attributed not only to its anti-hypertensive action per se, but also to the quantitative, as well as qualitative alterations of the lipid profile.

MetS is characterized by atherogenic dyslipidemia consisting of elevated serum triglycerides and apoB levels, in- creased small dense LDL particles, and reduced levels of HDL-C [1,2,15-17]. Small dense LDL particles are the most atherogenic LDL particles and represent a risk factor for coronary artery disease, independently of serum LDL-C levels $[16,18,19]$. ApoB levels denote the number of atherogenic lipoproteins in circulation [20-23], and some investigators believe that total apoB is the best target of lipidlowering therapy [20,22-24]. In this particular setting of dyslipidemia, the unique properties of zofenopril, such as the existence of the - $\mathrm{SH}$ group and its action on nitric oxide (NO) may be important for the reduction of apolipoprotein B levels, as well as for the predominance of large LDL over small dense LDL particles. There are limited data on the effect of other ACE inhibitors on lipoprotein metabolism with temocapril hydrochloride reported to normalise the LDL particle size [25]. Moreover, zofenopril exerts antioxidant activity beyond its well-known anti-hypertensive action. In particular, zofenopril decreases systemic oxidative stress in hypertensive patients, estimated by the reduction of plasma and LDL hydroperoxides, plasma 8-isoprostanes, circulating oxidized-LDL, and asymmetrical dimethyl-Larginine (ADMA), which is a competitive inhibitor of endo- 
thelial NO synthase, compared to non-SH containing ACE inhibitors $[10,11]$. Moreover, zofenopril increases flow mediated dilation (FMD), which is a reliable method for the evaluation of endothelial function and reflects NO availability $[10,11,26]$. Thus, through its antioxidant activity, zofenopril offers important advantages in reducing endothelial activation and retarding vascular dysfunction $[10,11]$. In our study, zofenopril additionally altered the lipid profile, while there were no major changes in the weight or the waist circumference, and the patients had specific dietary instructions. Moreover, the reduction of small dense LDL particles was strongly correlated with the reduction in triglyceride levels. This finding is explained by the fact that serum triglyceride concentration is one of the most important determinants of the presence of small dense LDL particles [27]. This dual effect of zofenopril (the antioxidant activity and the improvement in lipid profile), whether interconnected or independent, may be particularly important for this subset of patients with MetS. There are no previous reports on the effect of zofenopril on triglyceride levels, but reductions on triglyceride levels have been reported with other angiotensin receptor enzyme inhibitors, explained by the fact that the alteration in AT1 receptor density reduces the plasma lipids $[28,29]$. On the other hand, triglyceride levels are not stable metabolic parameters and they are easily influenced by a variety of factors like variations in dietary habits, even though all patients had taken specific instructions for their diet. Furthermore, the population of this study had metabolic syndrome, and zofenopril affected the lipid profile in patients characterized by high triglyceride levels.

Lp-PLA2, an enzyme mainly associated with LDL, is currently related to the inflammatory mechanisms of the atherosclerotic process. Lp-PLA2 exhibits a $\mathrm{Ca}^{++}$ independent phospholipase A2 activity and catalyzes the hydrolysis of the ester bond at the sn-2 position of the proinflammatory phospholipid mediator platelet activating factor. Lp-PLA $A_{2}$ also degrades oxidatively fragmented phospholipids, which are formed during the oxidative modification of LDL and are implicated in atherogenesis [30,31]. However, it should be noted that the role of Lp-PLA $\mathrm{P}_{2}$ remains controversial, since unanswered questions still exist with respect to this enzyme and its direct biologic role in atherosclerosis [32]. Zofenopril decreased Lp-PLA 2 activity, contrary to other anti-hypertensive medications [33]. This effect of zofenopril on Lp-PLA $\mathrm{P}_{2}$ activity could be explained by the fact that the activity of this LDL-associated enzyme is mainly distributed on small dense LDL particles [30,34], which were reduced with zofenopril.

Zofenopril did not influence glucose homeostasis, with no significant alterations of glucose and insulin levels, as well as the HOMA index. Although there is no specific data on the effect of zofenopril on glucose homeostasis, a metaanalysis reported a protective role of long term use of other ACE inhibitors for the development of diabetes mellitus [35]. Moreover, data from the DREAM (Diabetes REduction Assessment with ramipril and rosiglitazone Medications) trial showed that among persons with impaired fasting glucose levels or impaired glucose tolerance, the use of ramipril for 3 years did not reduce the incidence of diabetes, but increased regression to normoglycemia [36].
In the present study, zofenopril did not alter fibrinogen levels. There are no available data concerning the effect of zofenopril on fibrinogen levels, though fibrinogen remained unchanged following the administration of other ACE inhibitors $[37,38]$.

Currently, guidelines focus on blood pressure levels as the main target of therapy and zofenopril seems to achieve the desirable blood pressure levels. Moreover, in this particular group of individuals with MetS, zofenopril favourably influenced some of the lipid parameters related to increased atherogenicity. This promising finding derives from a distinctive drug with special properties among the ACE inhibitors and deserves further research taken into account the limitations of a non randomised study, with a small number of patients like this one. Further studies are needed to confirm the above results.

\section{CONCLUSION}

Zofenopril is an anti-hypertensive agent that seems to have additional favourable effects on the atherogenic dyslipidemia in patients with MetS.

\section{REFERENCES}

[1] Grundy, S.; Cleeman, J.I.; Daniels, S.R.; Donato, K.A.; Eckel, R.H.; Franklin, B.A.; Gordon, D.J.; Krauss, R.M.; Savage, P.J.; Smith, S.C. Jr.; Spertus, J.A.; Costa, F. Diagnosis and management of the metabolic syndrome: an American Heart Association/National Heart, Lung, and Blood Institute Scientific Statement. Circulation, 2005, 112, 2735-52.

[2] Reilly, M.P.; Rader, D.; The metabolic syndrome. Circulation, 2003, 108, 1546-51.

[3] Athyros, V.G.; Ganotakis, E.S.; Elisaf, M.S.; Liberopoulos, E.N.; Goudevenos, I.A.; Karagiannis, A. Prevalence of vascular disease in metabolic syndrome using three proposed definitions. Int. J. Cardiol., 2007, 117, 204-10.

[4] Eckel, R.H.; Grundy, S.M.; Zimmet, P.Z. The metabolic syndrome. Lancet, 2005, 365, 1415-28.

[5] Grundy, S.M. Metabolic syndrome: a multiplex cardiovascular risk factor. J. Clin. Endocrinol. Metab., 2007, 92, 399-404.

[6] Gami, A.S.; Witt, B.J.; Howard, D.E.; Erwin, P.J.; Gami, L.A.; Somers, V.K.; Montori, V.M. Metabolic syndrome and risk of incident cardiovascular events and death: a systematic review and meta-analysis of longitudinal studies. J. Am. Coll. Cardiol., 2007, 49, 403-14.

[7] Liberopoulos, E.N.; Elisaf, M.S. Diagnosis of the metabolic syndrome: which definition should we use? Hellenic J. Cardiol., 2005, 46, 258-62.

[8] Athyros, V.G.; Ganotakis, E.S.; Bathianaki, M.; Monedas, I.; Goudevenos, I.A.; Papageorgiou, A.A.; Papathanasiou, A.; Kakafika, A.I.; Mikhailidis, D.P.; Elisaf, M. Awareness, treatment and control of the metabolic syndrome and its components: a multicentre Greek study. Hellenic J. Cardiol., 2005, 46, 380-6.

[9] Ambrosioni, E.; Borghi, C.; Magnani, B. for the survival of myocardial infarction long-term evaluation (smile) study investigators: The effect of the angiotensin-converting-enzyme inhibitor zofenopril on mortality and morbidity after anterior myocardial infarction. N. Engl. J. Med., 1995, 332, 80-5.

[10] Pasini, A.F.; Garbin, U.; Nava, M.C.; Stranieri, C.; Pellegrini, M.; Boccioletti, V.; Luchetta, M.L.; Fabrizzi, P.; Lo. Cascio, V.; Cominacini, L. Effect of sulfhydryl and non-sulfhydryl angiotensinconverting enzyme inhibitors on endothelial function in essential hypertensive patients. Am. J. Hypertens., 2007, 20, 443-50.

[11] Napoli, C.; Sica, V.; de. Nigris, F.; Pignalosa, O.; Condorelli, M.; Ignarro, L.J.; Liguori, A. Sulfhydryl angiotensin-converting enzyme inhibition induces sustained reduction of systemic oxidative stress and improves the nitric oxide pathway in patients with essential hypertension. Am. Heart J., 2004, 148(5), e5.

[12] Koh, K.K.; Han, S.H.; Quon, M.J. Inflammatory markers and the metabolic syndrome: insights from therapeutic interventions. J. Am. Coll. Cardiol., 2005, 46, 1978-85. 
[13] Paoletti, R.; Gotto, A.M. Jr.; Hajjar, D.P. Inflammation in atherosclerosis and implications for therapy. Circulation, 2004, 109, 1206.

[14] Tselepis, A.D.; Dentan, C.; Karabina, S.A.; Chapman, M.J.; Ninioc, E. PAF-degrading acetylhydrolase is preferentially associated with dense LDL and VLDL-1 in human plasma: catalytic characteristics and relation to the monocyte-derived enzyme. Arterioscler. Thromb. Vasc. Biol., 1995, 15, 1764-73.

[15] Ayyobi, A.F.; Brunzell, J.D. Lipoprotein distribution in the Metabolic Syndrome, type 2 diabetes mellitus, and familial combined hyperlipidemia. Am. J. Cardiol., 2003, 9, 27-33.

[16] Gazi, I.F.; Tsimihodimos, V.; Tselepis, A.D.; Elisaf, M.; Mikhailidis, D.P. Clinical importance and therapeutic modulation of small dense low-density lipoprotein particles. Expert Opin. Biol. Ther., 2007, 7, 53-72.

[17] Brunzell, J.D.; Ayyobi, A.F. Dyslipidemia in the metabolic syndrome and type 2 diabetes mellitus. Am. J. Med. 2003, 115, 24-8.

[18] Carmena, R.; Duriez, P.; Fruchart, J.C.; Atherogenic lipoprotein particles in atherosclerosis. Circulation, 2004, 109, III2-7.

[19] Kwiterovich, P.O. Jr. Clinical relevance of the biochemical, metabolic, and genetic factors that influence low-density lipoprotein heterogeneity. Am. J. Cardiol., 2002, 90, 30-47.

[20] Sniderman, A.D. Applying apoB to the diagnosis and therapy of the atherogenic dyslipoproteinemias: a clinical diagnostic algorithm. Curr. Opin. Lipidol., 2004, 15, 433-8.

[21] Yusuf .S.; Hawken, S.; Ounpuu, S.; Dans, T.; Avezum, A.; Lanas, F.; McQueen, M.; Budaj, A.; Pais, P.; Varigos, J.; Lisheng, L. Effect of potentially modifiable risk factors associated with myocardial infarction in 52 countries (the INTERHEART study): casecontrol study. Lancet, 2004, 364, 937-52.

[22] Pischon, T.; Girman, C.J.; Sacks, F.M.; Rifai, N.; Stampfer, M.J.; Rimm, E.B. Non-high-density lipoprotein cholesterol and apolipoprotein B in the prediction of coronary heart disease in men. Circulation, 2005, 112, 3375-83.

[23] Brunzell JD. Increased apo B in small dense LDL particles predicts premature coronary artery disease. Arterioscler. Thromb. Vasc. Biol., 2005, 25, 474-5.

[24] Sattar, N.; Williams, K.; Sniderman, A.D.; D’Agostino, R. Jr.; Haffner, S.M. Comparison of the associations of apolipoprotein B and non-high density lipoprotein cholesterol with other cardiovascular risk factors in patients with the metabolic syndrome in the Insulin Resistance Atherosclerosis Study. Circulation, 2004, 110, 2687-93.

[25] Miyashita, Y.; Ito, Y.; Hasiguchi, S.; Totsuka, M.; Aoki, A.; Kurokawa, M.; Tomioka, H.; Shirai, K. Effect of temocapril hydrochloride on serum lipid levels in patients with hypertensive type 2 diabetes mellitus. J. Atheroscler. Thromb., 2001, 8, 5-9.

[26] Corretti, M.C.; Anderson, T.J.; Benjamin, E.J.; Celermajer, D.; Charbonneau, F.; Creager, M.A.; Deanfield, J.; Drexler, H.; Gerhard-Herman, M.; Herrington, D.; Vallance, P.; Vita, J.; Vogel, R. Guidelines for the ultrasound assessment of endothelialdependent flow-mediated vasodilation of the brachial artery: a report of the International Brachial Artery Reactivity Task Force. $J$. Am. Coll. Cardiol., 2002, 39, 257-65.
[27] Gazi, I.; Tsimihodimos, V.; Filippatos, T.; Bairaktari, E.; Tselepis, A.D.; Elisaf M: Concentration and relative distribution of lowdensity lipoprotein subfractions in patients with metabolic syndrome defined according to the National Cholesterol Education Program criteria. Metabolism, 2006, 55, 885-91.

[28] Kowala, M.; Grove, R.; Aberg, G. Inhibitors of angiotensinconverting enzyme decrease early atherosclerosis in hyperlipidemic hamster. Fosinopril reduces plasma cholesterol and captopril inhibits macrophage-foam cell accumulation independently of blood pressure and plasma lipids. Atherosclerosis, 1994, 108, 61-72.

[29] Gaudio, G.; Guasti, L.; Schizzarotto, A.; Simoni, C.; Crespi, C.; Cimpanelli, M.; Klersy, C.; Grandi, A.M.; Rigandi, G.; Venco, A. Changes in plasma lipids during renin-angiotensin system blockade by combination therapy (enalapril plus valsartan) in patients with diabetes and hypertension. J. Cardiovasc. Pharmacol., 2005, 45, 362-366.

[30] Rizos, E.; Tambaki, A.P.; Gazi, I.; Tselepis, A.D.; Elisaf, M. Lipoprotein-associated PAF-acetylhydrolase activity in subjects with the metabolic syndrome. Prostaglandins Leukot. Essent. Fatty Ac$i d s, \mathbf{2 0 0 5}, 72(3), 203-9$.

[31] Tselepis, A.D.; Chapman, J.M. Inflammation, bioactive lipids and atherosclerosis: potential roles of a lipoprotein-associated phospholipase A2, platelet activating factor-acetylhydrolase. Atheroscler Suppl., 2002, 3, 57-68.

[32] Zalewski, A.; Nelson, J.J.; Hegg, L.; Macphee, C. Lp-PLA2: a new kid on the block. Clin. Chem., 2006, 52(9), 1645-50.

[33] Tambaki, A.P.; Rizos, E.; Tsimihodimos, V.; Tselepis, A.D.; Elisaf. M. Effects of antihypertensive and hypolipidemic drugs on plasma and high-density lipoprotein-associated platelet activating factor-acetylhydrolase activity. J. Cardiovasc. Pharmacol. Ther., 2004, 9, 91-5.

[34] Tsimihodimos, V.; Karabina, S.A.P.; Tambaki, A.P.; Bairaktari, E.; Goudevenos, J.A.; Chapman, M.J.; Elisaf, M.; Tselepis, A.D. Atorvastatin preferentially reduces LDL-associated plateletactivating factor acetylhydrolase activity in dyslipidemias of type IIA and type IIB. Arterioscler. Thromb. Vasc. Biol., 2002, 22, 30611.

[35] Andraws, R.; Brown, D.L. Effect of inhibition of the reninangiotensin system on development of type 2 diabetes mellitus (meta-analysis of randomized trials). Am. J. Cardiol., 2007, 99, 1006-12.

[36] Bosch, J.; Yusuf, S.; Gerstein, H.C.; Pogue, J.; Sherigan, P.; Dagenais, G.; Diaz, R.; Avezum, A.; Lanas, F.; Probstfield, J.; Fodor, G.; Holman, R.R. Effect of ramipril on the incidence of diabetes. $N$. Engl. J. Med., 2006, 355, 1551-62.

[37] Spencer, C.G.; Felmeden, D.C.; Blann, A.D.; Lip, G.Y. Effects of "newer" and "older" antihypertensive drugs on hemorrheological, platelet, and endothelial factors. A substudy of the AngloScandinavian Cardiac Outcomes Trial. Am. J. Hypertens., 2007, 20, 699-704.

[38] Fogari, R.; Mugellini, A.; Zoppi, A.; Corradi, L.; Preti .P.; Lazzari, P.; Derosa, G. Losartan and perindopril effects on plasma plasminogen activator inhibitor-1 and fibrinogen in hypertensive type 2 diabetic patients. Am. J. Hypertens., 2002, 15, 316-20.

(C) Rizos et al.; Licensee Bentham Open.

This is an open access article licensed under the terms of the Creative Commons Attribution Non-Commercial License (http://creativecommons.org/licenses/ by-nc/3.0/) which permits unrestricted, non-commercial use, distribution and reproduction in any medium, provided the work is properly cited. 